\title{
Consequências do comportamento sedentário no bem-estar psicossocial: estudo qualitativo com idosos residentes em Portugal \\ Consequences of sedentary behavior on psychosocial well-being: a qualitative study with older adults living in Portugal
}

\section{Consecuencias del comportamiento sedentario en el bienestar psicosocial: un estudio} cualitativo con personas mayores que viven en Portugal

\author{
*André Ramalho, * João Petrica, *João Serrano, *Rui Paulo, *Pedro Duarte-Mendes, **António Rosado \\ *Instituto Politécnico deC astelo Branco (Portugal), ${ }^{* *}$ U niversidadedeLisboa(Portugal)
}

\begin{abstract}
Resumo.Asevidênciascientíficassugerem queo excesso decomportamento sedentárioéum fator derisco paraasaúdefíicadosidosos, independentemente dos seus níveis de atividade física. No entanto, o conhecimento acerca das consequências do comportamento sedentário em diversos indicadores psicossociaisnapopulação idosa, está, ainda, pouco explorado. 0 objetivo do estudo foi identificar ecompreender asconsequênciasdo comportamento sedentário no bem-estar psicossocial deidosos residentesem Portugal. Participaram no estudo 14 idososcom idades compreendidasentre 0565 e 0573 anos. Comafinalidadededescrever osparticipantesentrevistados, utilizou-sequestionáriosquepermitiram estimar otempo médio diário utilizado em diferentes comportamentossedentárioseo nível habitual deatividadefísica. Além disso, os dadosforam recolhidosatravésdeentrevistassemiestruturadas eanalisadosem função dos procedimentos daanálisetemática. Foi identificado queasdiferentes dimensõesdo comportamento sedentário (o tipo de comportamento sedentário, ainterrupção do comportamento sedentário, o tempo ininterrupto sentado eafrequência) podem ter influênciano bemestar psicossocial dosidosos Além disso, foram identificadostrêstemas (aperceção demelhoriaedemanutenção defunções cognitivas, aperceção de estados afetivos positivos e a interação social percebida) associados à promoçãa do bem-estar psicossocial dos idosos e dois temas (a perceção da diminuiç̧̃̃o derelaç̃oessociaiseaperceção desintomasdefadigamental) quepodem contribuir paraadeterioração do mesmo. 0 sresultadosdesteesudo são úteisparaacompreensão daexperiênciadosidososem relação a comportamento sedentário easimplicaçõesdestenadimensão cognitiva, emocional esocial, dassuasvidas.
\end{abstract}

Palavras-chaves tempo sentado, indicadorespsicossociais, envelhecimento, investigação qualitativa.

\begin{abstract}
Scientific evidencesuggeststhat excessivesedentary behavior isarisk factor for physical heatthin older adults, regardless oftheir activity level. However, knowledgeabout the consequences of sedentary behavior on variouspsychosocial indicatorsin theolder population isstill poor. Theaim of this studywasto identify and understand the consequences of sedentary behavior on thepsychosocial well-being of older adultsin Portugal. Fourteen older people, aged between 65 and 73 years, participted in the study. Q uestionnaireswere used to determinetheaveragedaily time spentin different sedentary behaviors and the usual level of physical activity. Datawere also collected through semi-structured interviews and analyzed using thematic analysis techniques. It was found that the different dimensions of sedentary behavior (type of sedentary behavior, interruption of sedentary behavior, time of uninterrupted sitting, and thefrequency) may influencethepsychosocial well-being of older adults. In addition, threethemes (perceived improvement and maintenance of cognitivefunction, perceived positive affectivestates, and perceived social interaction) wereassociated with promoting psychosocial well-beingin older adults, and two themes (perceived decreased social relationshipsand perceived symptoms of mental fatigue) may contributeto the dedinein psychosocial well-being Thefindingsof thisstudy areuseful for understandingolder adults' experiences of sedentary behavior anditsimpact on the cognitive, emotional, and social dimensions of their lives.
\end{abstract}

Keywords sedentarytime, psychosocial indicators, aging, qualitativeresearch

Resumen. Laspruebascientíficassugieren queun comportamiento sedentario excesivo esunfactor deriesgo paralasalud físicadel aspersonæmmayores, independientementedesusniveles deactividad física. Sin embargo, el conocimiento sobrelasconsecuenciasdel comportamiento sedentario en varios indicadores psicosociales en la población de edad avanzada está todavía poco explorado. El objetivo del estudio era identificar y comprender las consecuenciasdel comportamiento sedentario en el bienestar psicosocial delaspersonasmayoresresidentesen Portugal. Catorcepersonasmayoresde entre65 y 73 añosparticiparon en el estudio. Paradescribir alosparticipantes entrevistados, se utilizaron cuestionariosparaestimar el tiempo medio diario dedicado adiferentes comportamientos sedentariosy el nivel habitual deactividad física. Además, losdatosserecogieron medianteentrevistas semiestructuradas y se analizaron según los procedimientos del análisistemático. Seidentificó que las diferentes dimensiones del comportamiento sedentario (el tipo de comportamiento sedentario, lainterrupción del comportamiento sedentario, el tiempo ininterrumpido sentado y lafrecuencia) pueden tener unainfluenciaenel bienestar psicosocial delaspersonasmayores Además, seidentificaron trestemas (percepción demejoray mantenimiento delasfuncionescognitivas, percepción deestados afectivos positivosy percepción deinteracción social) asociadosalapromoción del bienestar psicosocial en las personas mayores y dos temas (percepción de disminución de las relaciones sociales y percepción de síntomas de fatiga menta) que pueden contribuir a su deterioro. Los resultados de este estudio son útiles para comprender la experiencia de las personas mayores en relación con el comportamiento sedentario y susimplicacionesen lasdimensionescognitiva, emocional y social desusvidas.

Palabrasclave: tiempo sentado, indicadorespsicosociales, envejecimiento, investigación cualitativa.

Fecha recepción: 29-11-20. Fecha de aceptación: 20-03-21

André Ramalho

andre.ramalho@ ipcb.pt 


\section{Introdução}

A investigação de Morris, Heady, Raffle e Parks (1953) foi pioneira ao demonstrar que os indivíduos fisicamente ativos apresentavam melhores resultados de saúde, em relação aos indivíduos que manifestavam excesso de comportamento sedentário diário. A terminologia atual define comportamento sedentário como aqueles comportamentos de vigília carderizados por um gasto energético ? a1, 5 equival entes metabólicos (METs) quando se está na posição sentada, reclinada ou deitada (Tremblay et al., 2017). U mavez que a redução do comportamento sedentário é proposta nas recomendações de prática de atividade física, o termo inativo fisicamente surgiu como mais adequado, em vez do termo sedentário, para classificar os indivíduos que não cumprem as recomendações de atividade física de intensidade moderada a vigorosa (Sedentary Behaviour Research N etwork, 2012). Desta forma, é importante entender que o conceito de comportamento sedentário é distinto do conceito de inatividade física e que as consequências para a saúde que resultam do excesso de comportamento sedentário são distintas daquelas que dizem respeito à inatividade física (O wen et al., 2017). Nos últimos anos, o estudo acerca das consequências do comportamento sedentário nos diferentes segmentos populacionais tem merecido atenção e as investigações têm sido orientadas pelo modelo da epidemiologia comportamental (Sallis \& O wen, 1999). Este modelo tem sido articulado para o estudo das consequências do comportamento sedentário na saúde biopsicossocial e, também, para a investigação dos determinantes do comportamento sedentário ( 0 wen, Healy, Matthews, $\&$ Dunstan, 2010).

0 comportamento sedentário representa grande parte do quotidiano da população idosa, uma vez que este segmento populacional real iza diferentes atividades sedentárias durante 9.4 horas/ dia (Harvey, Chastin, \& Skelton, 2015). Verificase a existência de um aumento de risco de mortalidade de $28 \%$ para aqueles idosos que assistem 5 ou maishoras/ diade televisão (Keadle, Arem, Moore, Sampson, \& Matthews, 2015). Além disso, a partir de um estudo de revisão sistemática de literaura foi possivel concluir a existência de associações significativas entre 0 excesso de comportamento sedentário diário e diferentes fatores cardiometabólicos, como a obesidade e a síndrome metabólica, dos idosos (de Rezende, Rey-López, Matsudo, \& do Carmo Luiz, 2014). No mesmo sentido, outras pesquisas epidemiológicas identificaram que o excesso de comportamento sedentário diário é um importante fator de risco para a saúdebiopsicossocial dosidosos, independentementedos seus níveis de atividade física (Chau et al. , 2013; Thorp, 0 wen, Neuhaus, \& Dunstan, 2011).

Relativamente às consequências psicossociais do comportamento sedentário, as evidências científicas publicadas mostram que determinadoscomportamentos sedentários podem ser positivos paraa saúde psicossocial dosidosos (M cewan,Tam-Seto, \& Dogra, 2016; 0 'N eill $\&$ Dogra, 2016). No entanto, outros resultadossugerem que o comportamento sedentário contribui para a deterioração da qualidade de vida do idoso (Balboa C astillo, León-M uñoz, Graciani, Rodríguez-Artalejo, \& Guallar-Castillón, 2011) e para a sintomatologia depressiva do idoso (Hamer, Poole, \& Messerli-Bürgy, 2013). Desta forma, o conhecimento sobre as consequências do comportamento sedentário em diversos indicadores psicossociais está, ainda, pouco explorado sendo necessária mais investigação (Copeland et al., 2017).

Especificamente no caso dos idosos, considerado o segmento mais sedentário da população, existe uma acentuadacarência de estudos de natureza interpretativa relacionados com as consequências psicossociais do comportamento sedentário (0 wen, 2017; Ramalho, Petrica, \& Rosado, 2018). Além disso, quando os baixos índices de prática de atividade física de intensidade moderada a vigorosa que a população idosa manifesta são combinados com o excesso de comportamento sedentário diário (Colley et al., 2011), significa que o estudo sobre as consequências do comportamento sedentário no que respeitaàsfunções cognitivas, sintomas depressivos e qualidade de vida no geral, é de particular importância (Dogra et al., 2017). 0 bem-estar psicossocial assume uma especial relevância para a saúde dosidosos, umavez quese encontrafortemente associado à longevidade e à diminuição de doenças cardíacas (Steptoe, Deaton, \& Stone, 2015). Parao propósito deste trabalho, o bem-estar psicossocial é considerado um constructo multidimensional, englobando as caraterísticas pessoais (valores, atitudes, crençass), os diversos estados emocionais, as relações sociais, e as funções cognitivas (por exemplo, a fluência verbal, velocidade de perceção, e a memória de trabalho) (Ekkekakis\& Backhouse, 2009).

0 excesso de comportamento sedentário diário é um fator de risco modificável e a sua redução é importante para se alcançar um envelhecimento saudável (Dogra\& Stathokostas, 2012). A investigação acerca das consequências do comportamento sedentário pode ser 
de particular interesse para os investigadores e para os diversos profissionais de intervenção geriátrica. N esse sentido, descrever as perceções dosidososacerca daquilo que pensam sobre o seu comportamento sedentário, poderá contribuir parao desenvolvimento de estratégias que permitam uma redução do sedentarismo e permitir 0 aumento dos níveis habituais de atividade física desta população. N outra direção, o presente estudo também poderá apresentar utilidade no contexto da sugestão de novas investigações, a partir do modelo da epidemiologia comportamental (0 wen et al., 2010), acerca das consequências do comportamento sedentário na saúde, em particular sobre os aspetos psicossociais. Desta forma, o objetivo deste estudo foi identificar e compreender as consequências do comportamento sedentário no bem-estar psicossocial de idosos residentes em Portugal.

\section{M étodo}

Este estudo foi conduzido a partir de uma perspetiva construtivista, procurando compreender os contextos socioculturais representados pelos participantes deste estudo (Charmaz, 2017), assumindo-se uma ontologia relativista, isto é, a existência de múltiplas perceções da realidade social (Guba $\&$ Lincoln, 2005). Assim, 0 presente estudo utilizou entrevistas semiestruturadas por permitem obter uma imagem de um conjunto de crenças ou perceções dos entrevistados, acerca de um tópico em particular (Smith, 1995). Escolheu-se esta abordagem uma vez que existem lacunas na literatura, no âmbito dos estudos qualitativos, acerca das consequências psicossociais do comportamento sedentário na população idosa. É adequada a utilização de uma abordagem qualitativa quando o conhecimento acerca de um determinado objeto de estudo, ainda, carece de explicação (Powell \& Single, 1996).

\section{Participantes}

0 critério de seleção dos participantes foi baseado na idade, a partir dos 65 anos. Relativamente aos critérios de exclusão teve-se em conta os seguintes: doença terminal e apresentar alterações motoras e sensoriais que limitem a realização de atividades físicas diárias. N esse sentido, os participantes foram incluídos com base nas respostas aos itens 1, 3, 4 e 5 da Composite Physical Functioning Scale (Rikli \& J ones, 2013); apenas aqueles que responderam "Consegue fazer por conta própriaesem ajuda" foram incluídosnaanálise. A seleção dos participantes foi realizada através de um processo intencional e a partir de uma estratégia de amostragem teórica (Coyne, 1997). A estratégia de amostragem teve lugar durante a recolha e a análise de dados. 0 processo envolveu a localização de novos casos de forma a permitir contrastar as perceções com os participantes previz mente selecionados. Assim, cada entrevistado foi selecionado a partir da análise de dados das entrevistas anteriores, permitindo construir uma heterogeneidade nas caraterísticas dos participantes. Portanto, procurouse uma estratégia de amostragem que permitisse garantir que determinadas caraterísticas, dentro de um universo de amostragem, pudessem ser representadas numa amostra final. Além disso, a partir da saturação teórica dos dados (Robinson, 2014), a recolha de dados foi interrompida, não tendo sido considerado 0 recrutamento de novos participantes. Foram recrutados catorze idosos (M édia = 67 anos deidade; Desvio padrão $=1.4$ ), sendo sete dos participantes do género feminino e sete do género mascul ino. 0 s por menores acerca das características dos participantes podem ser consultados na tabela 1.0 s participantes assinaram o consentimento informado de acordo com a Declaração de Helsínquia para estudos com humanos.

\section{Recolha de dados}

Numa primeira fase foram solicitadas algumas informações sociodemográficas aos participantes incluindo: a idade, o género, o local de habitação, a nacionalidade, o nível educacional, a profissão anterior, a raça/ etnia, e o estado civil. Posteriormente, os participantes foram avaliados através do questionário deVan Cauwenberg, Holle, Bourdeudhuij, 0 wen e Deforche (2014), no sentido de determinar o tempo total sedentário durante um dia normal. Este questionário permitiu que os idosos relatassem o tempo sedentário nosseguintesitens: a) visual ização de televisão ou vídeo/ DVD; b) utilização de computador; c) ler; d) socializar com amigos ou familiares; e) conduzir veícul os motorizados ou utilizar transportes públicos; f) realizar passatempos na posição sentada (por exemplo, jogos de tabuleiro); g) quaisquer outras atividades sedentárias. 0 nível de atividade física foi verificado através do questionárioYale Physical Activity Survey, validado para a população idosa portuguesa (M achado et al., 2016). Estes dados foram recolhidos com a finalidade de descrever melhor os participantes entrevistados.

Posteriormente, os dados foram recolhidos através de entrevistas individuais mediante um guião de entrevista semiestruturada. 0 guião da entrevista foi desenvolvido de acordo com os procedimentos de 
investigação interpretativa (Biddle, Markland, Gilbourne, Chatzisarantis, \& Sparkes, 2001). Desta forma, o guião de entrevista foi elaborado a partir das sugestões de M cewan et al. (2016) e analisado por dois investigadores independentes, com experiência em investigação qualitativa. Decorrente dessa análise, 0 conteúdo do guião de entrevista foi alvo de ligeiras alterações. Depois, com dois idosos selecionados por conveniência, foi realizado um pré-teste, com reflexão falada, no sentido de avaliar a adequação dos itens do guião de entrevista. A pós a real ização do pré-teste foram concretizadas modificações mínimas no conteúdo das questões do guião de entrevista semiestruturada.

As entrevistas seguiram uma abordagem gradual de três níveis de perguntas (perguntas do geral para o específico) (Tong et al., 2007). 0 guião da entrevista semiestruturada incluiu as seguintes questões de partida: "Q ual é a atividade que mais gosta de fazer quando está sentado? E a que menos gosta?"; "Q ue significado tem de estar sentado na sua vida diária?"; "Descreva os benefícios das atividades sedentárias que realiza diaria mente"; "D escreva os malefícios das atividades sedentárias que realiza diariamente"; "Prefere assistir televisão sozinho ou com companhia?"; "0 que sente quando está muito tempo sentado?"; "0 que o faz levantar quando está muito tempo sentado?"; "Q uando está a assistir televisão, realiza outra atividade sedentária ao mesmo tempo?";"Q uais as razões pelas quais você acha que os outros indivíduos não participam nas atividades sedentárias em que você participa? "Além disso, existiram outrasquestõesque foram desenvolvidas a partir dos dados emergentes, isto é, as respostas dos participantes induziram as questões subsequentes (Fletcher \& Sarkar, 2012). A recolha de dados ocorreu nas habitações de cada participante. No caso dos entrevistados que se encontravam institucionalizados, as mesmas ocorreram num espaço privado, cedido pela instituição. As entrevistas decorreram através de uma conversa informal, sendo registadas através de um gravador de áudio. No momento da realização das entrevistas apenas estavam presentes 0 entrevistado e 0 primeiro autor. Foi realizada uma entrevista com cada participante com uma duração média de 45 minutos.

\section{Análise de dados}

0 conteúdo das entrevistas foi transcrito na íntegra para formato digital e depois foi analisado através da análise temática (Braun \& Clarke, 2006), que incluiu a familiarização com os dados, a definição de códigos iniciais, a classificação dos códigos em temas, a revisão e refinamento dos temas e, finalmente, a redação da análise. 0 processo seguiu uma abordagem indutiva, na qual os temas foram identificados a partir dos dados recolhidos. A pesar de os dados terem sido analisados de forma indutiva, é preciso considerar que a construção das interpretações dos dados, foi, também, realizada a partir de uma perspetiva hermenêutica. N esse sentido, osinvestigadoresnão iniciam um estudo sem a existência de um conhecimento prévio acerca de uma determina da área de investigação (Weed, 2009). Assim, os conhecimentosteóricos, dos autores do presente estudo, foram utilizados com sensibilidade, sem serem impostos aos dados, permitindo garantir que os dados das entrevistas realizadas produzissem perspetivas únicas.

De forma a conferir credibilidade aos dados, 0 primeiro autor discutiu as suas interpretações com os coautores que tiveram a função de "amigo crítico" (Holt \& Sparkes, 2001). Assim, durante o curso de investigação foram realizadas seis reuniões de balanço, com toda a equipa de investigação, para analisar criticamente 0 processo de análise de dados. Os estudos adquirem credibilidade ao ser procurada a revisão por pares (Creswell \& Miller, 2000). As reuniões de balanço permitiram que o primeiro autor pudesse verificar se as suas interpretações pareciam plausíveis em relação aos dados e ajudaram no desenvolvimento de novas perspetivas em relação aos dados e às análises realizadas. O utra técnica utilizada foi a verificação participante (Lincoln \& Guba, 1985). N esse sentido, com o objetivo de avaliar a adequação dos temas emergentes dos dados, foram real izadas reuniões entre o primeiro autor e os entrevistados. As observações dos participantes entrevistados foram tomadas em consideração na interpretação dos dados. Desta forma, os temas emergentes dos dados foram desenvolvidos tendo em conta a sua exclusividade e exaustividade.

\section{Resultados}

O s participantes do presente estudo manifestam caraterísticas heterogéneas em relação ao nível educacional (variou desde 0 ensino básico até ao ensino superior), à experiência profissional (foram incluídos idosos que tiveram profissões fisicamente desgastantes (por exemplo, operário fabril) e outros idosos cuja profissão era realizada, maioritariamente, a partir da posição sentada (por exemplo, secretária), a diferentes locais de habitação (foram incluídos idosos institucional izados e idosos que vivam nas suas residências) e diferentes na cionalidades (doze participantes são de nacionalidade 
portuguesa e dois participantes são naturais da África do Sul). Desta forma, a amostragem de participantes com características diferenciadas poderá permitir esclarecer os aspetos críticos do fenómeno em estudo, contribuindo para asuageneralização parcial (Firestone, 1993).

O s participantes estão em média 532.8 minutos por dia ( $\mathrm{min} / \mathrm{d}$ ) em comportamentos sedentários. Relativamente à média de tempo que os participantes utilizam na realização de diferentes atividades sedentárias foram obtidos os seguintes resultados: visualização de tel evisão ou vídeo/ DVD (212 min/ d); utilização de computador (30 min/ d); ler (111 min/ d); socializar com amigos ou familiares (90 $\mathrm{min} / \mathrm{d})$; conduzir veículos motorizados ou utilizar transportes públicos (24 min/ d); realizar passatempos na posição sentada (por exemplo, jogos de tabuleiro) (60 $\mathrm{min} / \mathrm{d}$ ); quaisquer outras atividades

Tabela 1

\begin{tabular}{|c|c|c|c|c|c|c|c|c|c|c|}
\hline Participan & $\begin{array}{l}\text { sidade } \\
\text { (anos) }\end{array}$ & $\begin{array}{l}\text { énero } \\
\text { F/ M) }\end{array}$ & $\begin{array}{l}\text { Local de } \\
\text { habitaçãao }\end{array}$ & País & $\begin{array}{c}\text { Nível } \\
\text { educacional }\end{array}$ & Profissão anterior & $\begin{array}{l}\text { Raçal } \\
\text { etnia }\end{array}$ & $\begin{array}{c}\text { Estado } \\
\text { civil }\end{array}$ & $\begin{array}{l}\text { Comportamentc } \\
\text { sedentário } \\
(\mathrm{min} / \mathrm{d})\end{array}$ & $\begin{array}{l}\text { OAtividade física } \\
\text { (Suficientemen } \\
\text { te ativo) }\end{array}$ \\
\hline 1 & 68 & M & Casa própria & Portugal & Secundário & Motorista & Branca & Casado & 520 & Não \\
\hline 2 & 69 & $\mathrm{~F}$ & Casa própria & Portugal & Secundário & Secretária & Branca & Viúvo & 470 & Não \\
\hline 3 & 67 & $\mathrm{~F}$ & Casa própria & Portugal & Secundário & Doméstica & Branca & Casado & 530 & Não \\
\hline 4 & 65 & $\mathrm{~F}$ & Casa própria & Africa do Sul & Secundário & Cabeleireira & Branca & Casado & 520 & Não \\
\hline 5 & 66 & M & Casa própria & Africa do Sul & Básico & O perário fabril & Branca & Casado & 540 & Não \\
\hline 6 & 67 & M & Casa própria & Portugal & Licenciatura & Professora & Branca & Casado & 550 & Não \\
\hline 7 & 69 & $\mathrm{~F}$ & Casa própria & Portugal & Secundário & Funcionária pública & Branca & Casado & 550 & Não \\
\hline 8 & 71 & $\mathrm{~F}$ & Lar & Portugal & Básico & Serviço de limpeza & Branca & Casado & 530 & Não \\
\hline 9 & 68 & $\mathrm{~F}$ & Casa própria & Portugal & Licenciatura & Enfermeira & Branca & Viúvo & 530 & Não \\
\hline 10 & 66 & $\mathrm{~F}$ & Casa própria & Portugal & Licenciatura & Professora & Branca & Casado & 540 & Não \\
\hline 11 & 67 & M & Casa própria & Portugal & Secundário & Bombeiro & Branca & Casado & 530 & Não \\
\hline 12 & 73 & M & Lar & Portugal & Básico & 0 perário fabril & Branca & Viúvo & 560 & Não \\
\hline 13 & 66 & M & Casa própria & Portugal & Doutoramento & Engenheiro civil & Branca & Casado & 540 & Não \\
\hline 14 & 68 & M & Casa própria & Portugal & Secundário & Futebolista profissional & Branca & Casado & 550 & Não \\
\hline
\end{tabular}

sedentárias (5 min/ d). Relativamente à atividade física de intensidade leve os participantes utilizam, em média, $126 \mathrm{~min} / \mathrm{d}$. Em relação à atividade física de intensidade moderada a vigorosa verifica-se que os participantes realizam, em média, $15 \mathrm{~min} / \mathrm{d}$. Através da análise temática foi possível apresentar os conceitos que formam cada um dos temas e a frequência com que cada tema foi referenciado pelos idosos no decorrer das entrevistas. Todosos conceitos emergentes dos dadosforam agrupados por cada tema (por exemplo, raciocínio, concentração, memória, aprendizagem, reflexão e pensar diferente, foram associados ao tema perceção de melhoriae demanutenção de funções cognitivas) (Tabela 2). Da análise e interpretação dos discursos emergiram cinco temas os quais se associam em três dimensões psicossociais: a dimensão cognitiva, emocional e social, tal como é apresentado na figura 1. De seguida são apresentados os temas e utilizados extratos dos discursosdos participantes para melhor esclarecer o conteúdo desses temas.

\section{Perceção de melhoria e de manutenção de funções cognitivas}

O s dados sugeriram que muitosdosidosos entrevistados

Tabela 2

Temas emergentes dos dados

\begin{tabular}{|c|c|c|}
\hline Temas & Conceitos associados & Comportamentos Sedentários \\
\hline $\begin{array}{l}\text { Perceção de melhoria } \\
\text { e de manutenção de } \\
\text { funções cognitivas ( } F= \\
64 ; n=14 \text { ) }\end{array}$ & $\begin{array}{l}\text { Raciocínio, concentração, } \\
\text { memória, aprendizagem, } \\
\text { reflexão, pensar diferente. }\end{array}$ & $\begin{array}{l}\text { Realizar passatempos na posição } \\
\text { sentada (por exemplo, sudoku, } \\
\text { palavras cruzadas), leitura. }\end{array}$ \\
\hline $\begin{array}{l}\text { Perceção de estados } \\
\text { afetivos positivos ( } \mathrm{F}= \\
58 ; n=14 \text { ) }\end{array}$ & $\begin{array}{l}\text { Satisfação com a vida, sentir-se } \\
\text { bem, distração, felicidade, prazer, } \\
\text { relaxamento. }\end{array}$ & $\begin{array}{l}\text { Visual ização deTV, leitura, escrever } \\
\text { e-mails, utilização de redes sociais, } \\
\text { sentados em repouso. }\end{array}$ \\
\hline $\begin{array}{l}\text { Perceção de interação } \\
\text { social }(F=54 ; n=14)\end{array}$ & $\begin{array}{l}\text { Vida familiar, amigos, conver sar, } \\
\text { estabelecer novas relações sociais, } \\
\text { estar em casa, estar fora de casa. }\end{array}$ & $\begin{array}{l}\text { Visual ização deTV, sentados em } \\
\text { conver sa social. }\end{array}$ \\
\hline $\begin{array}{l}\text { Perceção de sintomas } \\
\text { de fadiga mental ( } F= \\
40 ; n=14)\end{array}$ & $\begin{array}{l}\text { Longos períodos inter ruptos } \\
\text { sentados, tensão, cansaço mental, } \\
\text { saúde mental, rotina, excesso de } \\
\text { atividade intelectual, inter romper } \\
\text { o comportamento sedentário. }\end{array}$ & $\begin{array}{l}\text { Visual ização deTV, real izar } \\
\text { passatempos na posição sentada } \\
\text { (por exemplo, jogos de tabul eiro), } \\
\text { leitura. }\end{array}$ \\
\hline $\begin{array}{l}\text { Perceção da } \\
\text { diminuiçã̃o de relações } \\
\text { sociais ( } F=33 ; n=14)\end{array}$ & $\begin{array}{l}\text { Longos períodos inter ruptos } \\
\text { sentados, necessidade de interagir } \\
\text { socialmente, solidão, estar em } \\
\text { casa, interromper o } \\
\text { comportamento sedentário. }\end{array}$ & $\begin{array}{l}\text { Visualização deTV, sentados em } \\
\text { repouso. }\end{array}$ \\
\hline
\end{tabular}

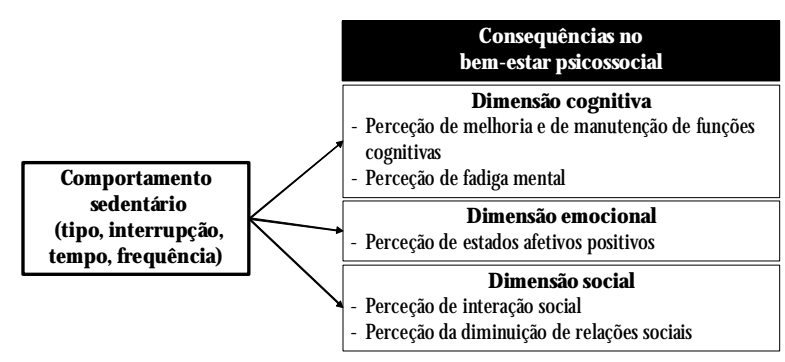

Figura 1. Consequências do comportamento sedentário no bem-estar psicossocial dos idosos percecionaram que a realização de certas atividades (por exemplo, sudoku, palavras cruzadas) possibilitavam manter edesenvolver acapacidade de raciocínio: "Ajuda me nas ligações entre as coisas.. . ligações de raciocínio. Desenvolve o meu raciocínio e a concentração." (Participante 9). Além disso, outros idosos eram da opinião que essas atividades sedentárias promoviam benefícios naconcentração: "0 sudoku eas palavras cruzadas ajuda me na concentração no diazadia" (Participante 5). A leituratambém foi mencionada como umaoportunidade para desenvolver pensamentos reflexivos, sendo considerada uma atividade fundamental no dia-a-dia dealguns idosos: "O ler transmite outro tipo de pensamentos... E a pensar em coisas, que antes, nunca tínhamos pensa do sequer. Eu acho que para mim a leitura é essencial. (Participante 10).

\section{Percecão de estados afetivos positivos}

Os dados sugeriram que a utilização de tecnologias de informação e das redes sociais proporcionava divertimento aos idosos que as utilizavam: "E então, eu divirto-me a fazer os e-mails, ou a responder, ou a 
receber. E a ver o facebook." (Participante 7). Noutro sentido, alguns dos entrevistados percecionaram que quando a vida diária não permitia estarem longos períodos sentados, o comportamento sedentário possibilitava relaxamento: "Sinto-mebem, quer dizer... com estas atividades porque para além da vida doméstica, estes bocadinhos quando estou a ver TV estou a dar um bocadinho de descanso à cabeça." (Participante 3). O utros participantesconsideraram que a leituraera considerada uma atividade prazerosa e por isso, era atribuído um significado de elevada importância na rotina diária: "Traz-me, essencialmente, a sensação de estar viva. Estou sentada, mas não estou!" (Participante 10).

\section{Perceção de interação social}

O s dados sugeriram que o comportamento sedentário poderia ser importante no estabelecimento de relações sociais dos idosos. N esse sentido, al guns participantes indicaram: "Também acho que verTV é bom para ser social, para comentar com o meu marido, os meus filhos, os meus netos até. $E$ até perguntar o que é que quer dizer aquilo." (Participante 6). Além disso, outros idosos consideraram que estarem sentados, em ambientes sociais como jardins públicos, em conversa com os seus amigos, constituíam-se ocasiões para discutirem assuntos dos seus interesses e ainda estabelecerem novas relações sociais: "Porque estar sentado no jardim, durante umas horas, a falar com os meus amigos é positivo. Há diálogo, o que é positivo (... ) e também pode permitir conhecer pessoas diferentes" (Participante 3).

\section{Perceção da diminui ção de relações sociais}

No presente estudo percebe-se, pelos discursos dos participantes, que o tempo prolongado de comportamento sedentário se associa a uma menor interação social. Nesse sentido, os entrevistados manifestaram que o longo período ininterrupto em comportamento sedentário, dentro das suas habitações, significava sentirem-se isolados: "Sinto que preciso de falar com al guém. Por exemplo, posso estar sentadauma hora ou duas a ver TV. Mas vem um momento qual quer que a gente... que tenho necessidade de ir a falar com alguém." (Participante 2). Para contrariar o isolamento, al guns dos idosos indicaram a necessidade de sair das suas habitações na procura de contacto social: "E depois se a gente se levantar, convivemos com aquele, com 0 outro... A nossa mente trabal ha e estamos mais leves. Agora, se estivermos em casa a descansar, isso não acontece... " (Participante 4).

\section{Perceção de fadiga mental}

0 excesso de tempo em atividades sedentárias foi mencionado como um fator que contribuía para a fadiga mental de alguns idosos: "Às vezes, quando estou a ler, parece que estou noutro mundo. Então tenho de interromper, levantar-me para voltar ao mundo real porque já estou cansada mentalmente". (Participante 10). Desta forma, al guns idosos percecionaram que 0 "Excesso de atividade intelectual é mau, porque depois a pessoa chega a ficar cansada mentalmente." (Participante 4). No entanto, os dados do presente estudo também sugeriram que os idosos sentiam a necessidade de se movimentarem, devido aos sintomas de fadiga mental, interrompendo os seus comportamentos sedentários para posteriormente retomálos: "Porque, acho que o meu organismo está a precisa de se movimentar. Sinto-me stressada, mentalmente cansada e tenho necessidade de fazer uma quebra e depois voltar a sentar-me" (Participante 6). Noutro sentido, outrosidosostinham a perceção que o excesso de tempo de atividades sedentárias passivas do ponto de vista cognitivo (por exemplo, ver TV), também originavam sintomas de fadiga: "Não me sinto bem. Chego até ao ponto de estar muito tempo sentada, até me canso. Sinto um cansaço mental." (Participante 2 ).

\section{Discussão}

Este estudo procurou identificar e estabelecer uma compreensão acerca das consequências do comportamento sedentário no bem-estar psicossocial de uma população idosa residente em Portugal. Os resultados sugerem que o tipo de comportamento sedentário, ainterrupção do comportamento sedentário, o tempo ininterrupto sentado e a frequência com que os idosos realizam uma determinada atividade sedentária, constituem-se variáveis importantes para a compreensão do impacto do comportamento sedentário no bem-estar psicossocial. Estas variáveis estão em concordância com as caraterísticas do comportamento sedentário (frequência, interrupção, tempo e tipo) que devem ser consideradas na relação entre o sedentarismo e a saúde (Tremblay, Colley, Saunders, H ealy, \& O wen, 2010). De facto, os estudos mais recentes começam a considerar a análise das diferentes dimensões do comportamento sedentário, como o tipo de comportamento sedentário (Hallgren et al., 2018), a interrupção e o volume de tempo ininterrupto sentado e a sua frequência (Sörman, Ljungberg, \& Rönnlund, 2018). 
Relativamente ao tipo de comportamento sedentário, é proposto queestadimensão seja classificada em duas categorias: os comportamentos sedentários mentalmente ativos e os comportamentos sedentários passivos. Desta forma, considera-se que os comportamentos sedentários mental mente ativos são aqueles que possibilitam a estimulação positiva do ponto de vista cognitiva, social e emocional (Hallgren et al., 2018) e os comportamentos sedentários passivos aqueles que não possibilitam uma estimulação significa tiva, pela positiva, nas dimensões referidas anteriormente (Kikuchi et al., 2014). De facto, as evidências anteriores mostram que os comportamentos sedentários passivos estão associadosaíndices mais baixosnas funções cognitivas dos idosos (Hamer \& Stamatakis, 2014), enquanto os comportamentos sedentários ativos encontram-se associados a mel horias no bem-estar psicossocial dos idosos (0 'Neil \& Droga, 2016). No entanto, existem numerosos comportamentos sedentários com características muito diferenciadas pelo que não seria coerente considerar que os benefícios ou os malefícios psicossociais resultantes do comportamento sedentário, sejam exatamente os mesmos. Nesse sentido, também é necessário considerar outras especificidades do tipo de comportamento sedentário, como o seu conteúdo. Assim, ver um jogo desportivo naTV poderá ter um impacto diferente, mais positivo ou menos positivo, no bem-estar psicossocial por comparação a assistir ao noticiário, em função dos inter esses pessoais dos idosos.

0 s resultados do presente estudo também sugerem que o comportamento sedentário, em função das diferentes dimensões enunciadas, pode proporcionar a melhoria e manutenção de funções cognitivas, estados afetivos positivos, interação social e diminuição de relações sociais, e, finalmente, sintomas de fadiga mental.

O s idosos participantes neste estudo percecionaram que determinados comportamentos sedentários possibilitavam a manutenção e a melhoria de diferentes indicadores cognitivos (por exemplo, memória semântica, memória de trabalho, raciocínio), o que vai ao encontro de outros estudos. Desde uma perspetiva neurobiológica, as atividades intelectuais ativam a utilização de redes neuronais que prol ongam a eficiência do sistema nervoso central ao longo da vida e, em particular, na população idosa (Slegers, van Boxtel, \& Jolles, 2006). Há evidências de que a realização de comportamentos sedentários ativos durante 40 a 60 minutos pode melhorar os aspetos cognitivos dos idosos
(Tranter \& Koutstaal, 2008). No mesmo sentido, os resultadosanterioresmostram que osidososqueutilizam o computador por maisde umal horapor diaapresentam melhorias na memória semântica e nas funções executivas por comparação com os idosos que não utilizam (K esse-Guyot et al., 2012). Desta forma, pa rece que os comportamentos sedentários mentalmente ativos possuem características cognitivamente estimulantes, podendo compensar a sua natureza passiva, do ponto de vista físico, em relação ao seu impacto no envelhecimento cerebral. No entanto, é preciso considerar que os comportamentos sedentários mental mente ativos podem apresentar diferentes níveis de estimulação cognitiva. Isto significa que as atividades sedentárias que apresentam caraterísticas mais superficiais do ponto de vista cognitivo são menos prováveis que envolvam a utilização de recursos cognitivos significativos (Sörman et al., 2018). Nesse sentido, para promover a saúde cognitiva dos idosos sugere-se uma redução do tempo em comportamentos sedentários passivos e 0 envolvimento em atividades físicas de intensidade moderada a vigorosa (AraqueMartínez, Ruiz-M ontero, \& Artés-Rodríguez, 2020; Astorga-Verdugo, Borges-Silva, González-Silva, Martínez-Araya, \& Rojas-Cabezas, 2020), assim como a realização de tarefas sedentárias cognitivamente estimulantes.

Os resultados também sugerem que 0 comportamento sedentário pode promover estados afetivos positivos (por exemplo, divertimento, humor e relaxamento). O s resultados aqui apresentados são consistentes com as evidências anteriores onde 0 comportamento sedentário pode satisfazer os vários aspetos das necessidades psicológicas de relaxamento (Ö stlund, 2010) e a melhorias no estado de humor dos idosos (0 'N eill, \& Dogra, 2016). Desta forma, 0 comportamento sedentário pode proporcionar uma experiência satisfatória, onde as oportunidades de ação de determinadas atividades sedentárias estão em equilíbrio com ascapacidades dosidosos. U ma explicação plausível acerca dos benefícios de determinadas atividades sedentárias nos estados afetivos positivos pode dever-se a estas poderem promover perceções de autonomia, deautoconfiança ede realização pessoal (Lee et al., 2017). Nesse sentido, a participação em tarefas sedentárias que os idosos considerem significativas podem estar associadas a uma maior satisfação e felicidade (Boyatzis, M cKee, \& Goleman, 2003). Assim, o tempo percebido dos idosos nos diferentes comportamentos sedentários pode ser influenciado pe- 
los valores, interesses e objetivos, assim como pelo nível de diversão e estimulação.

Noutra direção, os discursos dos participantes sugerem que o comportamento sedentário também pode ter uma influência positiva no estabelecimento das relações sociais do idoso. Desta forma, propõe-se que quando osidososque estão sentadosem ambientes sociais (por exemplo, jardins públicos), ou nas suas habitações, com as suas famílias ou amigos, constituíam-se em ocasiões para discutirem assuntos dos seus interesses e ainda estabelecerem novas relações sociais. De facto, as evidências anteriores mostram que o comportamento sedentário mentalmente ativo se encontra associado a uma maior interação social na população idosa (Cotten, Anderson, \& M cCullough, 2013; M cewan et al., 2016). Além disso, o comportamento sedentário que possibilita o contato social está associado a uma melhor saúde mental e a uma maior longevidade (Atkin, Adams, Bull, \& Biddle, 2012). Todavia, os dados do presente estudo também mostraram que 0 excesso de tempo em comportamento sedentário pode deteriorar a participação social do idoso, o que é corroborado por outros estudos. Por exemplo, a visualização deTV pode ser associada à solidão emocional dos idosos, apoiando a ideia que pode atuar como substituto dos contatos sociais mais próximos (Van der Goot, Beentjes, \& Van Selm, 2012). Assim, o excesso de tempo em comportamentos sedentários passivos pode estimular o isolamento social e limitar o desenvolvimento de redes de apoio social dos idosos (Golden et al., 2009). Desta forma, o risco para o isolamento social parece estar associado ao envolvimento em atividades sedentárias passivas, mas não para aquelas atividades sedentárias que são cognitivamente estimulantes, sugerindo que os contextos ambientaise sociais onde ocorrem o comportamento sedentário são importantes (H amer \& Stamatakis, 2014).

0 s resultados do presente estudo também sugerem que o demasiado tempo ininterrupto na realização de comportamentos sedentários, mentalmente ativos ou passivos, pode resultar em estados de fadiga mental para os idosos. N esse sentido, torna-se necessário distinguir a fadiga ativa, associada à sobrecarga cognitiva, da fadiga passiva, derivada da ausência de carga cognitiva e caraterizada pela monotonia (D esmond $\&$ Hancock, 2001). Desta forma, sugere-se que a realização de comportamentos sedentários mentalmente ativos e de comportamentos sedentários passivos podem, de forma distinta, influenciar o estado de fadiga mental dos idosos. Assim, parece plausível que as tarefas sedentárias passivas possam estar associadas a uma redução acen- tuada no interesse e na concentração dos idosos, resultando em fadiga passiva e a realização de tarefas sedentárias mental mente ativas resul tam em fadiga ativa (Matthews et al., 2010). Os dados dos entrevistados sugerem que ambas as formas de fadiga podem levar a queosidososinterrompam o comportamento sedentário. Assim, sugere-se que afadigamental pode ocorrer numa dimensão aguda (Shen, Barbera, \& Shapiro, 2006), uma vez que os idosos manifestaram que interrompem 0 comportamento sedentário por breves períodos para posteriormente retomá-lo. De facto, as pesquisas anteriores mostraram que as breves interrupções do comportamento sedentário, através de atividades físicas de baixa intensidade, atenuaram os níveis de fadiga depois dos indivíduos estarem sentados por um longo período ininterrupto (Wennberg et al., 2016).

0 excesso de comportamento sedentário diário tem consequências para a saúde dos idosos, distintas do impacto negativo da inatividade física (0 wen et al. , 2010). No entanto, para melhor compreender as associações entre o comportamento sedentário e os resultados de saúde biopsicossociais, torna-se necessário verificar os níveis de atividade física dos indivíduos em estudo (Doherty et al., 2017). De outra forma será difícil determinar se as associações com os resultados de saúde se devem ao excesso de comportamento sedentário diário ou à falta de prática de atividade física (Van der Ploeg \& Hillsdon, 2017). Além disso, o volume total da atividade física pode atenuar as associações entre 0 comportamento sedentário e os resultados de saúde (Maher, O Ids, Mire, \& Katzmarzyk, 2014). N esse sentido, o presente estudo avaliou o nível de atividade física e o comportamento sedentário com o objetivo de descrever melhor os participantes entrevistados. $0 \mathrm{~s}$ resultados deste estudo permitiram identificar que os idosos entrevistados manifestam baixos níveis de atividade física de intensidade moderada a vigorosa. Em termos do tempo utilizado em atividade física de intensidade moderada a vigorosa, os participantes deste estudo ficaram abaixo da recomendação de 150 minutos por semana de atividades física de intensidade moderada a vigorosa (Chodzko-Zajko et al., 2009). Os participantes do presente estudo também revelaram excesso de comportamento sedentário diário.

Estes resultados devem, também, ser considerados em relação ao bem-estar psicossocial dos idosos entrevistados, porque a prática de atividade física de intensidade moderada constitui-se numa medida eficaz, capaz de promover um impacto positivo na saúde psicossocial da população idosa, quando comparadacom 
o comportamento sedentário (Hogan, Catalino, Mata, $\&$ Fredrickson, 2015). N esse sentido, os resultados aqui apresentados podem contribuir para o desenvolvimento de intervenções que tenham como objetivo os idosos al cançarem um equilíbrio saudável entre o tempo utilizado em comportamentos sedentários, que pode ser benéfico para o bem-estar psicossocial, a prática de atividade física de baixa intensidade e a prática de atividade física de intensidade moderada a vigorosa, resultando numadiminuição do excesso de comportamento sedentário diário.

O s resultados deste estudo podem ser úteis para os diferentes profissionais que atuam em diferentes áreas da intervenção geriátrica (por exemplo, médicos, psicólogos, fisioterapeutas, técnicossuperiores de atividade física). 0 cansaço eafadigamental referidos pelosidosos, que os leva a alternar momentos de sedentarismo com atividade física, normalmente de baixa intensidade, e a necessidade de que expressam em sair do domicílio, parece transparecer uma vontade dos nossos participantes em diminuir o comportamento sedentário diário. É possível verificar que a literatura apresenta várias estratégias que podem ser úteis para os profissionais que atuam em contexto geriátrico e que pretendem apoiar os idosos a reduzir o seu comportamento sedentário diário.

Para possibilitar uma redução do comportamento sedentário diário dos idosos, os profissionais de intervenção geriátrica podem: encorajar os idosos a escolherem atividades sedentárias que permitam estímulos sociais, emocionais e cognitivos (ler, jogos de tabuleiro, puzzles, jogos de cartas, jogos de realidade virtual) (Campo-Prieto, Carral, O liveira, \& RodríguezFuentes, 2020), em detrimento das atividades sedentárias passivas; estruturar a prática de exercício físico, integrando, simultaneamente, a realização de tarefas cognitivas (Romero-Ramos, Romero-Ramos, \& González-Suárez, 2020; M artínez-Heredia, Santæella Rodríguez, \& Rodríguez-García, 2020), podendo ser uma forma de encorajar os idosos a substituírem as atividades sedentárias, realizadas diariamente, por mais atividades físicas de intensidade leve; desenvolver formas criativas de tornar certas atividades sentadas mais fisicamenteativas, permitindo desenvolver a autoeficácia dos idosos no que respeita à prática de exercício físico; estimular os idosos para o desenvolvimento de novas conexões sociais (participação em grupos comunitários que pratiquem regularmente exercício físico, participação nas universidades seniores, participação na organização de eventos de cariz solidário); incentivar os idosos à interrupção do comportamento sedentário durante os períodos de publicidade na televisão, realizando tarefas que exijam movimento. É, também, importante esclarecer os idosos de que a participação nos programas de atividade física pode não compensar os malefícios resultantes do excesso de comportamento sedentário realizado diariamente. Esta recomendação é relevante uma vez que os idosos podem justificar 0 comportamento sedentário através da realização de comportamentos ativos (por exemplo, prática de atividade física), manifestando as crenças de saúde compensatórias (Ramalho, Petrica, \& Rosado, 2019).

Este estudo permitiu alcançar al guns insights acerca das consequências psicossociais do comportamento sedentário dos idosos. No entanto, destaca-se uma limitação em relação às variáveis apresentadas, uma vez que, como quaisquer variáveis psicossociais, manifestam limitações inerentes, pois refletem as perceções dos participantes num momento específico e, portanto, estão sujeitas a mudança ao longo do tempo (Chatzisarantis \& Hagger, 2009). N esse sentido, sugere-se que, através da avaliação momentânea ecológica, as futuras investigações possam alcançar uma compreensão mais aprofundada, acerca dos consequentes psicossociais do comportamento sedentário. U ma outra limitação diz respeito à transferência dos resultados para outros contextos, diferenciados, daquele onde os idosos participantes dos estudos pertencem (região centro de Portugal). É preciso ter em consideração de que a vida quotidiana na cidade, conceptual izada como tendo um ritmo de vida mais ativo, é diferente da vida diária em zonas rurais, onde, por vezes, é fisicamente mais ativa. Desta forma, os futuros estudos podem recrutar, simultaneamente, participantes pertencentes a diferentes regiões (urbanas e rurais) e, se possível, países, de forma a potenciar a transferência dos resultados. Além disso, sugere-se que as futuras investigações possam avaliar determinadas condições patológicas dos idosos, como por exemplo o nível de dor crónica e a sintomatologia depressiva, uma vez que estas variáveis podem influenciar as perceções dos idosos acerca das consequências do comportamento sedentário. Não obstante, os resultados aqui apresentados podem generalizar-se pelo estudo em detalhe de um caso concreto, pela comparação com outros casos através da similitude proximal, aplicando-se uma lógica de replicação para contextos similares e pelo recurso à teoria. Além disso, para se estabelecer uma compreensão mais general iza da acerca das consequências do comportamento sedentário dos idosos, sugere-se que os estudos futuros 
tenham em consideração as diferentes dimensões do comportamento sedentário aqui apresentadas. Nesse sentido, a medição do comportamento sedentário pode ser realizada, concomitantemente, através de questionários e de acelerómetros. Os estudos futuros, também, podem considerar a análise de dados qualitativos e quantitativos, em simultâneo, através de métodos mistos, de forma a poder conhecer novas perspetivas sobre os aspetos psicossociais do quotidiano em relação ao comportamento sedentário do idoso.

\section{Conclusão}

0 s resultados do presente estudo permitiram concluir que os idosos entrevistados manifestavam excesso de comportamento sedentário diário, além de apresentarem baixos níveis de atividade física de intensidade moderada a vigorosa. Estes resultados devem considerados na compreensão das consequências psicossociais do comportamento sedentário pois destacam a necessidade do desenvolvimento de inter venções que permitam reduzir o comportamento sedentário diário. Além disso, este estudo permitiu identificar que as diferentes dimensões do comportamento sedentário (o tipo de comportamento sedentário, a interrupção do comportamento sedentário, o período ininterrupto sentado e a frequência com que os idosos realizam o comportamento sedentário) podem ter influência nas diferentes dimensões do bem-estar psicossocial dosidosos. Foram também identificadostrês temas (perceção de melhoria e de manutenção de funções cognitivas, perceção de estados afetivos positivos e a perceção de interação social) que podem possibilitar a promoção do bem-estar psicossocial dos idosos, edoistemas (perceção da diminuição de relações sociais e a perceção de sintomas de fadiga mental) que podem contribuir para a deterioração do mesmo. Estes resultados devem ser interpretados no contexto da literatura anterior, onde é evidenciado que o excesso de sedentarismo é prejudicial para a saúde biopsicossocial, aumentando o risco de mortalidade prematura dos idosos.

\section{Referências}

Araque-M artínez, M., Ruiz-M ontero, P. \& A ArtésRodríguez, E. (2020). Efectos de un programa de ejercicio físico multicomponente sobre la condición física, la autoestima, la ansiedad y la depresión de personasadultas-mayores. Retos, 39, 1024-1028. doi: https:/ / doi.org/ 10.47197/ retos. v0i39.83282

Astorga-Verdugo, S., Borges-Silva, F., González-Silva,

S. , Martínez-Araya, A., \& Rojas- Cabezas, G. (2020). Efectividad de un entrenamiento de fuerza con ca racterística social izadora y lúdica sobre los dominios de la calidad de vida en adultos mayores con anteposición de cabeza y cuello. Retos, 39, 713-717. doi: https:/ / doi.org/ 10.47197/ retos. v0i39.80316

Atkin, A., Adams, E. , Bull, F. , \& Biddle, S. (2012). Nonoccupational sitting and mental well-being in employed adults. Annals of Behavioral Medicine, 43(2), 181-188. doi: 10.1007/ s12160-011-9320-y.

Balboa-Castillo, T., León-M uñoz, L., Graciani, A., Rodríguez-Artalejo, F., \& Guallar-Castillón, A. (2011). Longitudinal association of physical activity and sedentary behavior during leisure time with health-related quality of life in community-dwelling older adults. Health and quality of life outcomes, 9, 1-10. doi: 10.1186/ 1477-7525-9-47

Biddle, S., Markland, D., Gilbourne, D., Chatzisarantis, N., \& Sparkes, A. (2001). Research methods in sport and exercise psychology: quantitative and qualitative issues. Journal of Sports Sciences, 19, 777-809. doi: 10.1080/ 026404101317015438

Boyatzis, R., McKee, A., \& Goleman, D. (2003). Reawakening your passion for work. Clinical leadership and management review, 17, 75-81.

Braun, V. \& Clarke, V. (2006) Using thematic analysis in psychology. Q ualitative Research in Psychology, 3, 77-101. doi: 10.1191/ 1478088706qp0630a

Campo-Prieto, P., Carral, J., O liveira, I., \& RodríguezFuentes, G. (2020). Realidad Virtual Inmersiva en personas mayores: estudio de casos. Retos, 39, 10011005. doi: https:// doi.org/ 10.47197/ retos. v0i39.78195

Charmaz, K. (2017). The Power of Constructivist Grounded Theory for Critical Inquiry. Q ualitative Inquiry, 23, 34-45. doi: 10.1177/ 1077800416657105

Chatzisarantis, N., \& Hagger, M. (2009). Effects of an inter vention based on self-determination theory on self-reported leisure-time physical activity participation. Psychology and Health, 24, 29-48.

Chau, J., Grunseit, A., Chey, T., Stamatakis, E., Brown, W., M atthews, C., ... Van der Ploeg, H. (2013). Daily sitting time and all-cause mortality: a meta-analysis. PLoS O ne, 8: e80000. doi: 10.1371/ journal. pone. 0080000 .

Chodzko-Zajko, W. J., Proctor, D. N., Fiatarone Singh, M. A., Minson, C. T., Nigg, C. R., Salem, G. J., \& Skinner, J. S. (2009). American College of Sports 
Medicine position stand. Exercise and physical activity for older adults. Medicine and science in sports and exercise, 41, 1510-1530. doi:10.1249/ MSS.0b013e3181a0c95c

Colley, R., Garriguet, D., Janssen, I., Craig, C., Clarke, J., \& Tremblay, M. (2011). Physical activity of Canadian adults: accelerometer resultsfrom the 2007 to 2009 Canadian Health M easures Survey. Health reports, 22, 7-14.

Copeland, J., A she, M., Biddle, S., Brown, W., Buman, M., Chastin, S., ... Dogra, S. (2017). Sedentary time in older adults: a critical review of measurement, associations with health, and interventions. British Journal of Sports M edicine, 51, 1539.

Cotten, S., Anderson, W., \& McCullough, B. (2013). Impact of internet use on loneliness and contact with others among older adults: cross-sectional analysis. Journal of medical Internet research, 15, e39. doi: 10.2196/ jmir. 2306.

Coyne, I. (1997). Sampling in qualitative research. Purposeful and theoretical sampling; merging or clear boundaries? Journal of Advanced N ursing, 26, 623-630.

Creswell, J.W., \& Miller, D.L. (2000) Determining Validity in Q ualitative Inquiry. Theory into Practice, 39, 124-130. http:/ / dx. doi.org/ 10.1207/ s15430421tip3903_2

de Rezende, L. F., Rey-López, J. P., Matsudo, V. K., \& do Carmo Luiz, O. (2014). Sedentary behavior and health outcomes among older adults: a systematic review. BMC public health, 14, 333. doi:10.1186/ 1471-2458-14-333

Desmond, P., \& Hancock, P. (2001). Active and passive fatigue states. In P. Desmond, \& P. Hancock (Eds.), Stress, workload, and fatigue (pp. 455-465). New Jersey: Erlbaum.

Dogra, S. \& Stathokostas, L. (2012). Sedentary behavior and physical activity are independent predictors of succesfful aging in middle-aged and older adults. Journal of Aging Research, 2012, 1-8. doi: 10.1155/ 2012/ 190654

Dogra, S., Ashe, M., Biddle, S., Brown, W., Buman, M., Chastin, S., ... Copeland, J. (2017). Sedentary time in older men and women: an international consensus statement and research priorities. British Journal of Sports Medicine, 51, 1526-1532. doi: 10.1136/ bjsports-2016-097209

Doherty, A., Jackson, D., Hammerla, N., Plötz, T., Olivier, P., Granat, M., ... Wareham, N. (2017). Large Scale Population Assessment of Physical
Activity U singW ristWorn Accelerometers: The UK Biobank Study. PLoS O ne, 12: e0169649. doi: 10.1371 / journal. pone. 0169649

Ekkekakis, P., \& Backhouse, S. (2009). Exercise and Psychological Well?Being. In R. J. Maughan (Ed.), O lympicTextbook of Science in Sport (pp. 251-271). 0 xford: W iley-Blackwell.

Firestone, W. (1993). Alternative arguments for generalizing from data as applied to qualitative research. Educational Researcher, 22, 16-23.

Fletcher, D., \& Sarkar, M. (2012). A grounded theory of psychological resilience in 0 lympic champions. Psychology of Sport and Exercise, 13, 669-678. doi:10.1016/ j. psychsport.2012.04.007

Golden, J., Conroy, R., Bruce, I., Denihan, A., Greene, E., Kirby, M., \& Lawlor, B. (2009). Loneliness, social support networks, mood and wellbeing in community-dwelling elderly. International journal of geriatric psychiatry, 24, 694-700. doi: 10.1002/ gps. 2181.

Guba, E., \& Lincoln, Y. (2005). Paradigmatic controversies, contradictions, and emerging con?uences. In N.K. Denzin \& Y.S. Lincoln (Eds.), The Sage handbook of qualitative research (pp. 191215). Thousand $O$ aks, CA: Sage.

Hallgren, M., O wen, N., Stubbs, B., Zeebari, Z., Vancampfort, D., Schuch, F., ... Lagerros, Y. (2018). Passive and Mentally-Active Sedentary Behaviors and Incident M ajor Depressive Disorder: a 13-Year Cohort Study. Journal of Affective Disorders, 241, 579-585. doi: 10.1016/ j.jad.2018.08.020

Hamer, M ., \& Stamatakis, E. (2014). Prospective Study of Sedentary Behavior, Risk of Depression, and Cognitive Impairment. Medicine and science in sports and exercise, 46, 718-723. doi: 10.1249/ MSS. 0000000000000156.

Hamer, M., Poole, L., \& Messerli-Bürgy, N. (2013). Television viewing, $\mathrm{C}$-reactive protein, and depressive symptomsin older adults. Brain, Behavior, and Immunity, 33, 29-32. doi: 10.1016/ j. bbi.2013.05.001.

Harvey, J., Chastin, S., \& Skelton, D. (2015). How sedentary are older people? A systematic review of the amount of sedentary behavior. Journal of aging and physical activity, 23, 471-487. doi: 10.1123/ japa. 2014-0164.

Hogan, C. L., Catalino, L. I., M ata, J., \& Fredrickson, B. L. (2015). Beyond emotional benefits: physical activity and sedentary behaviour affect psychosocial resources through emotions. Psychology \& health, 
30, 354-369. doi:10.1080/ 08870446.2014.973410

Holt, N., \& Sparkes, A. (2001). An ethnographic study of cohesiveness in a college soccer team over a season. The Sport Psychologist, 15, 157-172.

Keadle, S., A rem, H., Moore, S., Sampson, J., \& Matthews, C. (2015). Impact of changesin television viewing time and physical activity on longevity: a prospective cohort study. International Journal of Behavioral Nutrition and Physical Activity, 12, 156. doi: 10.1186/ s12966-015-0315-0

Kesse-Guyot, E., Charreire, H., Andreeva, V., Touvier, M., Hercberg, S., Galan, P., \& O ppert, J. (2012). Cross-Sectional and longitudinal associations of different sedentary behaviors with cognitive performance in older adults. PLOS ONE, 7: e47831. doi: 10.1371/ journal. pone. 0047831

Kikuchi, H., Inoue, S., Sugiyama, T., O wen, N., O ka, K., Nakaya, T., \& Shimomitsu, T. (2014). Distinct associations of different sedentary behaviors with health-related attributes among older adults. Preventive Medicine, 67, 335-339. doi: 10.1016/ j.ypmed. 2014.08.011

Lee, S., Ju, Y, Han, K., Choi, J., Yoon, H., \& Park, E. (2017). The association between loss of work ability and depression: a focus on employment status. International archives of occupational and environmental health, 90, 109-116. doi: 10.1007/ s00420-016-1178-7

Lincoln, Y., \& Guba, E. (1985). Naturalistic inquiry. Newbury Park, CA: Sage.

Machado, M., Tavares, C., M oniz-Pereira, V., André, H., Ramalho, F., Veloso, A., \& Carnide, F. (2016). Validation of YPAS-PT - TheYale Physical Activity Survey for Portuguese O Ider People. Science Journal of Public Health, 4, 72-80. doi: 10.11648/ j.sph. 20160401.20

Maher, C., Olds, T., Mire, E., \& Katzmarzyk, P. (2014). Reconsidering the Sedentary Behaviour Paradigm. PLOS ONE 9(1), e86403. doi:10.1371/ journal. pone. 0086403

Martínez-Heredia, N., Santaella-Rodríguez, E., \& Rodríguez-García, A. (2020). Beneficios de la actividad física para la promoción de un envejecimiento activo en personas mayores. Revisión bibliográica. Retos, 39, 829-834. doi: https:/ / doi.org/ 10.47197/ retos. v0i39. 74537

M atthews, G., Warm, J., Reinerman-Jones, L., Langheim, L., Washburn, D., \&Tripp, L. (2010). Task engagement, cerebral blood flow velocity, and diagnostic monitoring for sustained attention. J ournal of experimental psychology applied, 16, 187-203. doi: 10.1037/ 20019572.

M cewan, T., Tam-Seto, L., \& Dogra, S. (2016). Perceptions of Sedentary Behavior Among Socially Engaged O Ider Adults. The Gerontologist, 57, 735744. doi: 10.1093/geront/ gnv689

Morris, J., Heady, P., Raffle, C., \& Parks, J. (1953). Coronary heart disease and physical activity of work. Lancet, 2, 1053-1057.

0 'Neill, C., \& Dogra, S. (2016). Different Types of Sedentary Activities and Their Association W ith Perceived Health andWellnessAmong Middle-Aged and OIder Adults: A Cross-Sectional Analysis. American Journal of Health Promotion, 30, 314322. doi: $10.1177 / 0890117116646334$

Ö stlund, B. (2010). Watching television in later life: A deeper understanding of TV viewing in the homes of old people and in geriatric care contexts. Scandinavian Journal of Caring Sciences, 24, 233243. doi: 10.1111/ j.1471-6712.2009.00711.x

0 wen, N. (2017). Emergence of Research on Sedentary Behavior and Health. InW. Zhu \& N. O wen (Eds.), Sedentary behavior and health. Concepts, assessments, and interventions (pp. 3-12). Champaign, IL: Human Kinetics.

0 wen, N., Healy, G., Matthews, C., \& Dunstan, D. (2010). Too much sitting: the population health science of sedentary behavior. Exercise and Sport Science Reviews, 38, 105-113. doi: 10.1097/ JES.0b013e3181e373a2

Powell, R. A., \& Single, H. M. (1996). Focus groups. International Journal or Q uality in Health Care, 8, 499-504. doi: 10.1093/ intqhe/ 8.5.499

Ramalho, A., Petrica, J., \& Rosado, A. (2018). Sedentary behaviors and psychological outcomes among older adults: a systematic review. Motricidade, 14, 73-85. doi: 10.6063/ motricidade. 12223

Ramalho, A., Petrica, J., \& Rosado, A. (2019). Ascrenças de saúde compensatórias e o comportamento sedentário dos idosos: estudo qualitativo. Retos, 37, 264-272. doi: https:// doi.org/ 10.47197/ retos. v37i37. 71984

Rikli, R. E., \& Jones, C. J. (2013). Development and validation of criterion-referenced clinically relevant fitness standards for maintaining physical independence in later years. The Gerontologist, 53, 255-267. doi: 10.1093/ geront/ gns071

Robinson, O. (2014). Sampling in Interview-Based Q ualitative Research: A Theoretical and Practical Guide. Q ualitative Research in Psychology, 11, 25- 
41. doi: $10.1080 / 14780887.2013 .801543$

Romero-Ramos, N., Romero-Ramos, 0., \& GonzálezSuárez, A. (2020). Actividad física y funciones cognitivas en personas mayores: revisión sistemática de losúltimos 5 años. Retos, 39, 1017-1023. doi: https:/ / doi.org/ 10.47197/ retos. V0i39. 79960

Sallis, J., \& 0 wen, N. (1999). Physical activity and behavioral medicine. Thousand 0 aks, CA: Sage.

Sedentary Behaviour Research N etwork. (2012). Letter to the editor: Standardized use of the terms "sedentary" and "sedentary behaviours." Applied Physiology, Nutrition, and M etabolism, 37, 540-542. doi:10.1139/ h2012-024

Shen, J., Barbera, . . \& Shapiro, C. (2006). Distinguishing sleepiness and fatigue: focus on definition and measurement. Sleep medicine reviews, 10, 63-76.

Slegers, K ., van Boxtel, M., \& J olles, J. (2006). Successful cognitive aging: the use of computers and the Internet to support autonomy in later life. M aastricht: Neuropsych Publishers.

Smith, J. (1995). Semi-structured interviewing and qualitative analysis. In J. Smith, R. Harré, \& L. Van Langenhove (Eds.), Rethinking methods in psychology (pp. 9-26). London: Sage.

Sörman, D., Ljungberg, J., \& Rönnlund, M. (2018). Reading habits among older adultsin relation to level and 15-year changes in verbal fluency and episodic recall. Frontiers in psychology, 9: 1872. doi: 10.3389/ fpsyg 2018.01872.

Steptoe, A., Deaton, A., \& Stone, A. (2015). Psychological wellbeing, health and ageing Lancet, 385, 640-648. doi:10.1016/ S0140-6736(13)614890

Thorp, A., O wen, N., Neuhaus, M., \& Dunstan, D. (2011). Sedentary behaviors and subsequent health outcomes in adults: A systematic review of Iongitudinal studies. American Journal of Preventive Medicine, 41, 207-215. doi: 10.1016/ j. amepre.2011.05.004.

Tong, A., Sainsbury, P., \& Craig, J. (2007). Consolidated criteria for reporting qualitative research (COREQ): a 32-item checklist for interviews and focus groups. International Journal for Q uality in Health Care, 19, 349-357. doi: 10.1093/ intghc/ mzm042

Tranter, L., \& Koutstaal, W. (2008). Age and flexible thinking: an experimental demonstration of the beneficial effects of increased cognitively stimulating activity on fluid intelligence in healthy older adults. Neuropsychol Dev Cogn B Aging Neuropsychol
Cogn, 15,184-207. doi:10.1080/ 13825580701322

Tremblay, M., Aubert, S., Barnes, J., Saunders, T., Carson, V., Latimer-Cheung, A., ... SBRM Terminology ConsensusProject Participants. (2017). Sedentary Behavior Research N etwork (SBRN) Terminology Consensus Project process and outcome. International Journal of Behavioral N utrition and Physical Activity, 14: 74. doi: 10.1186/ s12966-017-0525-8.

Tremblay, M., Colley, R., Saunders, T., Healy, G., \& O wen, N. (2010). Physiological and health implications of a sedentary lifestyle. Applied Physiology, Nutrition, and M etabolism, 35, 725-740. doi: 10.1139/ H10-079.

Van Cauwenberg, J., van Holle, V., de Bourdeudhuij, I., O wen, N., \& Deforche, B. (2014). Older adult's reporting of specific sedentary behaviors: validity and reliability. BMC Public Health, 14:734. doi: 10.1186/ 1471-2458-14-734

Van der Goot, M., Beentjes, J., \& Van Selm, M. (2012). Meanings of television in older adults' lives: an analysis of change and continuity in television viewing. Ageing and Society, 32, 147-168. doi: 10.1017/ S0144686X 1100016X

Van der Ploeg, H., \& Hillsdon, M. (2017). Is sedentary behaviour just physical inactivity by another name? International Journal of Behavioral Nutrition and Physical Activity, 14:142. doi: 10.1186/ s12966-0170601-0

Weed, M. (2009). Research quality considerations for grounded theory research in sport \& exercise psychology. Psychology of Sport and Exercise, 10, 502-510. doi: 10.1016/ j. psychsport.2009.02.007

Wennberg, P., Boraxbekk, C., W heeler, M., Howard, B., Dempsey, P., Lambert, G., ... Dunstan, D. (2016). A cute effects of breaking up prolonged sitting on fatigue and cognition: a pilot stud. BMJ 0 pen, 6 : e009630. doi: 10.1136/ bmjopen-2015-009630

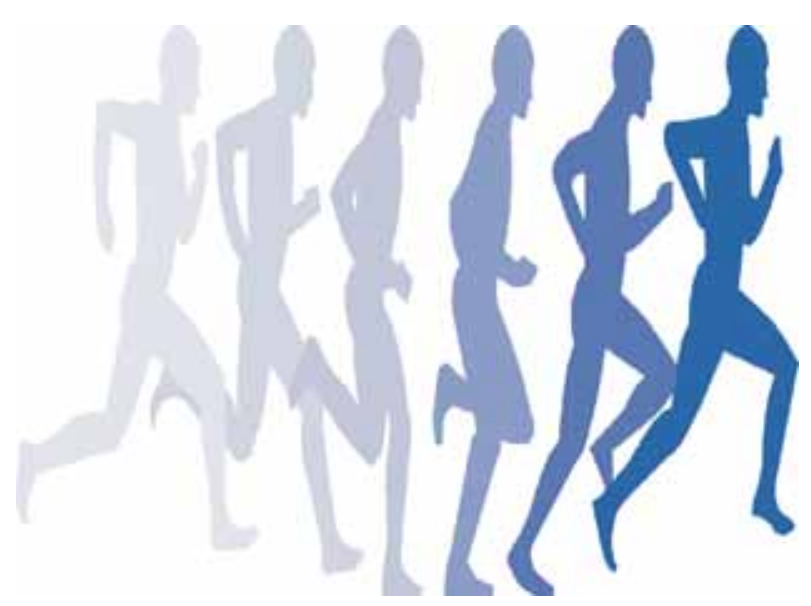

